\title{
L'exploitation des pâturages de savane en République Centrafricaine
}

\author{
par B. PEYRE DE FABREGUES (*)
}

\begin{abstract}
RÉSUMÉ
Grâce à des conditions de milieu relativement favorables, l'élevage des zébus introduit en R. C. A. il y a un demi-siècle environ, s'est remarquablement développé Mais l'accroissement du cheptel en contribuant à une importante dégradation de la végétation pastorale met en danger l'existence même du troupeau. Après avour examiné quelques-unes des contraintes pesant sur cet élevage, l'auteur propose diverses actions qui seraient à mettre en place sur les plans de la recherche et du développement pour tenter de sauvegarder le potentiel de l'élevage bovin en République Centrafricaine.
\end{abstract}

\section{GÉNÉRALITÉS}

L'élevage bovin est d'introduction assez récente sur le territoire de la République Centrafricaine. C'est, en effet, vers 1924, que des pasteurs peuls bororos sont venus s'installer au nordouest de Bouar, dans la région des plateaux alors pratiquement exempts de trypanosomose.

Un accroissement continu de leur cheptel, résultant principalement de l'abondance des ressources fourragères et des interventions du Service de l'Elevage dans le domaine sanitaire, conduisit rapidement les éleveurs à occuper une région de plus en plus vaste et, vers 1940 environ, détermina certains d'entre eux à aller se fixer dans un nouveau secteur situé dans le centre-est du pays, près de Bambari (cf. carte).

Actuellement, l'élevage bovin est largement développé dans ces 2 régions de la République Centrafricaine où, selon de récentes estimations, l'effectif total du cheptel zébu-bororo, atteindrait 800000 têtes environ. La zone occidentale, et particulièrement les savanes d'altitude de la région de Bouar, en abriterait 580000 environ : la zone centre-est, limitée à lorigine à un petit secteur touchant Bambari à l'est (mais qui s’est

(*) I. E. M. V. T., 10 rue Pierre-Curie, 94700 MaisonsAlfort, France. beaucoup étendu ces dernières années), en abriterait 220000 environ.

Les autres bovins, de races trypano-tolérantes, appartiennent généralement à des agriculteurs sédentaires. Répartis sur l'ensemble du territoire, leurs effectifs sont estimés de 15 à 20000 Baoulés et quelques centaines de N'Damas et de Lagunes.

Les deux régions principales d'élevage s'inscrivent dans le domaine du climat tropical humide qui n'est pas, traditionnellement une terre d'éleveurs mais d'agriculteurs-chasseurs. Les territoires actuellement exploités par les pasteurs peuls étaient "vides ", certes, à leur arrivée, mais non inexploités. Ce caractère de nouveauté de l'élevage aux yeux des sédentaires autochtones, accentué par l'absence de points communs entre les populations dès lors en présence, entre leurs traditions et leurs activités, a miné le contexte actuel de l'élevage en République Centrafricaine.

Des études réalisées jusqu'à ce jour, il ressort que la cohabitation entre agriculteurs et éleveurs, si elle pose des problèmes sur le plan de l'exploitation des territoires de chasse livrés à l'élevage extensif, est, en fin d'analyse, un facteur positif de développement. Elle est donc souhaitable. Par suite, les projets de programmes de développement devront s'orienter vers un 


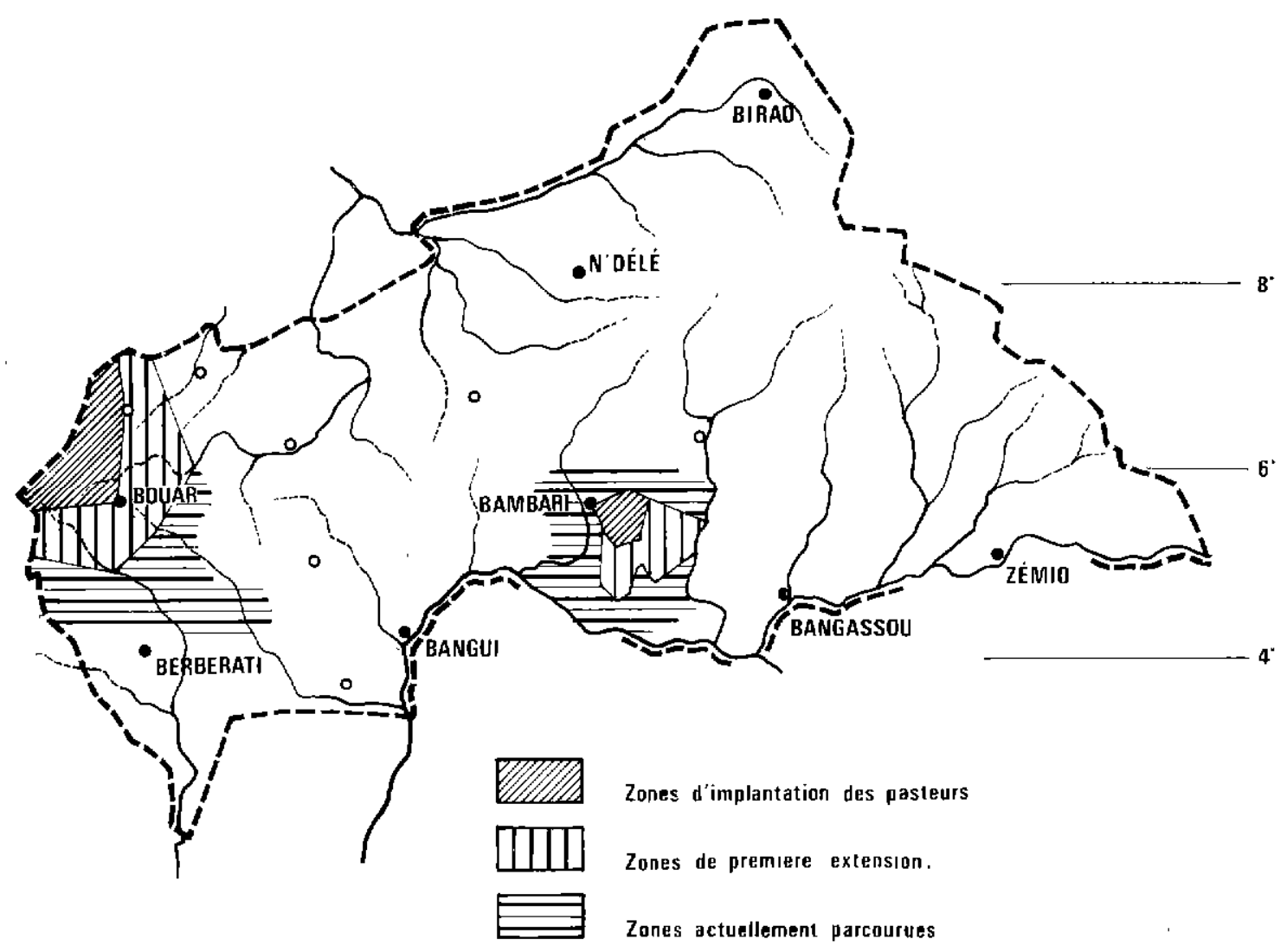

accroissement de la complémentarité des activités des agriculteurs et des éleveurs et non vers leur concurrence.

\section{Etat actuel des pâturages naturels en R. C. A.}

Le potentiel pastoral naturel de la République, représenté par la quasi-totalité des formations végétales possédant un tapis herbacé principalement graminéen, qui constituent les savanes développées sous climat tropical humide, est considérable.

Cependant, laccroissement du cheptel bovin associé à l'exploitation de la végétation par la libre pâture y ont abouti, particulièrement dans la zone occidentale, à la mise en route de processus d'évolution de la végétation correspondant à une diminution de la valeur fourragère.

La densification du couvert ligneux sur de grandes surfaces et la disparition de la végétation pâturable par place sont - avec la modification régressive (au sens fourrager) des couverts herbacés - les principales formes d'évolution du tapis végétal résultant du surpâturage qui provoquent la diminution du potentiel pastoral.

Sur le terrain, un certain nombre de symptômes (régression des espèces bonnes fourragères, mise à nu du sol, érosion, etc...) ou de plantes indicatrices (dont Panicum phragmitoides, Harungana madagascariensis, Arthrosamanea eriorachis sont parmi les plus importantes) révèlent l'apparition des processus régressifs.

Jusqu'ici, cette régression n'a guère retenti sur l'accroissement des effectifs du cheptel bovin parce que peu à peu les troupeaux ont migré en abandonnant les secteurs dégradés pour gagner de nouvelles savanes pâturables, situées plus bas tant en altitude qu'en latitude. Mais il semble que ce mouvement, favorisé par la conjoncture résultant des années de sécheresse, ne pourra pas durer encore longtemps.

En effet, d'une part, bien que très vastes, les savanes encore inexploitées par l'élevage sont tout de même limitées et, d'autre part, elles sont de plus en plus proches du domaine forestier. Or, l'augmentation de l'importance du couvert ligneux dans les pâturages de savane (qu'il résulte ou non de surpâturage) entraîne, outre une modification de la flore herbacée dans le sens d'une moindre valeur fourragère, l'accroissement du risque de trypanosomose.

La présence d'un bétail nombreux dans les zones hébergeant normalement des glossines risque de favoriser la pullulation des mouches 
et, par suite, la reconstitution du réservoir de trypanosomes. Les difficultés de constitution d'un stock de trypanocides et la dispersion des moyens d'intervention rendent cette situation inquiétante.

Par suite, on s'achemine probablement vers une ré-infestation par la mouche tsé-tsé des parcours actuellement pâturés. Si cela se produit, les troupeaux devront refluer s'ils ne veulent pas subir de sérieuses pertes. Or, un exode hors des frontières nationales n'est plus possible ; de sorte que le reflux du bétail ne pourrait se faire que vers les savanes les plus anciennement occupées, "exemptes " de tsé-tsé, mais dont la dégradation par suite de surpâturage a considérablement réduit le potentiel fourrager quand elle ne l'a pas anéanti... Naturellement, la " décharge " en bétail qui résulte de la dégradation a réalisé une sorte de mise en repos de la végétation. Cette "mise en défens" a autorisé une reprise des processus de régénération spontanée de la végétation. Mais cette évolution est extrêmement lente et, sous climat tropical humide, elle s'oriente plus vers la reconstitution de formations forestières que de savanes... le potentiel pastoral n'y gagne rien, bien au contraire.

Il est donc urgent de définir des modes d'exploitation et des techniques de régénération des pâturages naturels de savane susceptibles, selon le cas, d'augmenter, de conserver ou de réhabiliter le potentiel fourrager.

L'aménagement des zones de pâturage et l'amélioration des modes d'exploitation sont des mesures à mettre en œuvre rapidement.

De même, et malgré la réticence traditionnelle des Bororos devant cette éventualité, un plan de métissage du cheptel zébu avec des races trypano-tolérantes doit être élaboré si l'on veut se préparer à faire face aux difficultés auxquelles l'élevage risque de se trouver confronté à moyen terme.

\section{Amélioration des techniques de l'exploitation des parcours}

Dans un premier temps, une certaine amélioration des techniques d'exploitation des parcours semble pouvoir être rapidement obtenue par la mise en cuvre de mesures nouvelles très simples.

Par exemple, la protection du potentiel végétal pastoral et l'aménagement d'un calendrier fourrager éliminant les périodes de disette peuvent résulter, en grande partie, de la maî- trise des feux courants, de l'application d'un cycle de rotation incluant la mise en repos périodique des pâturages, et du respect d'un rapport d'équilibre entre charge et productivité végétale.

Dans la pratique, l'application sur le terrain de ce type de mesures se trouve entravée par un certain nombre de contraintes, dont l'élimination constitue un préalable à toute modification des techniques de l'élevage extensif.

\section{- Contraintes liées à la gestion}

Le système actuel d'exploitation des parcours de savane par le pastoralisme itinérant ne peut s'améliorer que s'il s'accompagne d'une discipline librement consentie et définie au moins au niveau de la communauté des éleveurs usufruitiers du même secteur. Les éleveurs Bororos doivent prendre conscience qu'en définitive, ils sont seuls maîtres de leur avenir en Centrafrique.

Or, le choc de l'économie moderne en ouvrani des perspectives à certains éléments des populations, en créant de nouveaux rapports de force, tend à détruire toute la discipline traditionnelle. Ainsi, les éleveurs de la nouvelle génération souhaitent souvent une modification radicale de leur mode de vie qui désarçonne les anciens et remet en cause leur autorité.

Entre populations différentes, ce phénomène est illustré au niveau des feux de brousse : allumés par les uns ou les autres, au seul vu de leurs intérêts immédiats, ils ne satisfont plus, correctement, ni à leur but de chasse ni à leur objectif pastoral qui est de provoquer la repousse.

Or, traditionnellement, la gestion des terrains de chasse, devenus des parcours, dépendait des sédentaires et elle leur a pratiquement échappé au profit des éleveurs, nouveaux venus...

Enfin, la propriété privée n'existe pas dans le secteur rural de l'Empire, au sens « européen ». La terre appartient à des groupes sociaux (villages, clans, tribus par exemple) et sa mise en valeur est organisée en fonction de décisions prises au niveau du groupe. En revanche, lexploitation est individuelle ou plus exactement familiale.

De sorte que, dans ce contexte, l'amélioration de la gestion des parcours nécessitera moins des investissements fonciers à réaliser que l'élaboration préalable d'un statut juridique constituant un « code rural " qu1, sans déposséder les agriculteurs, occupants traditionnels, assurera aux pasteurs, utilisateurs actuels, une 
pérennité d'usage suffisante pour qu'ils bénéficient des fruits de l'amélioration effectuée.

Cela doit être fait sans pour autant constituer un droit de propriété inaliénable au profit des éleveurs. Cet écueil serait insurmontable dans le contexte centrafricain où les agriculteurs se considèrent comme les propriétaires "indivis" de leurs terroirs de chasse, actuellement mis en valeur par l'élevage aux mains des peuls.

Réciproquement, il n'apparaît pas raisonnable qu'en vertu de ces mêmes droits traditionnels, les agriculteurs sédentaires s'opposent à l'exploitation, pour l'élevage, de secteurs largement sous-utilisés car réservés à la chasse.

\section{- Contraintes liées à la végétation}

Les plantes fourragères présentent divers degrés d'appétibilité, de sorte que la pâture, par le broutage sélectif, provoque le déséquilibre des compositions floristiques et aboutit, à plus ou moins long terme, à la disparition des espèces bonnes fourragères. Le fait que les troupeaux pâturent en général assez librement aggrave cette tendance, surtout pour les pâturages de saison des pluies.

En saison des pluies, en effet, le bétail est rassemblé, non loin du village où résident le berger ou le propriétaire, sur des surfaces relativement réduites. La libre pâture y est possible durant toute la saison grâce à la croissance continue de l'herbe et à la facilité de l'abreuvement au marigot voisin. Par goût, les animaux consomment de préférence les repousses de plantes récemment broutées, de sorte que c'est dans ces secteurs de stationnement en saison des pluies que s'observent les plus importants dégâts résultant du surpâturage.

En saison sèche, la ration est constituée par des repousses après feux peu volumineuses et ne croissant que très lentement, ce qui oblige le bétail à parcourir de grandes surfaces d'herbages. La possibilité de choisir et surtout de rebrouter la même plante s'en trouve réduite et, par conséquent, le danger d'élimination d"une espèce par surpâturage diminue beaucoup.

Il s"ensuit que l'importante différence de productivité des herbages entre saison sèche et saison des pluies entraîne des difficultés d'adaptation de la charge. Tant que le mode d'élevage restera extensif, qu'on n'envisagera donc pas de constituer des réserves fourragères pour la "saison de disette ", il faudra nécessairement exploiter en saison sèche des surfaces beaucoup plus grandes qu'en saison des pluies.

La pratique actuelle de la transhumance résulte, en grande partie, de cette nécessité. Les pâturages de saison des pluies, avoisinant les "villages " peuls, et saturés en bétail durant la période d'activité intense de la vie végétale, ne produisent plus, dès novembre, de quoi affourager les troupeaux.

De plus, l'absence de maîtrise des feux tant pour la date de l'incendie que pour l'importance des surfaces brûlées, entraîne une grande imprévisibilité de la capacité de charge des repousses de saison sèche et par suite contribue à la difficulté d'organisation de la transhumance.

Cependant, le problème résultant de la disparité des surfaces nécessaires d'une saison à l'autre n'est pas insoluble. Mais sa solution nécessite l'attribution éventuellement temporaire des droits d'exploitation à des groupes d'éleveurs bien définis et possesseurs d'un troupeau d'importance connue afin de les mettre face à leurs responsabilités. On en revient donc à un problème de gestion dépendant en particulier d'une modification des traditions concernant le droit à la terre...A ce propos, il faut noter que la loi 65-61 du 3 juin 1965 portant réglementation de l'élevage en République Centrafricaine précisait, entre autres, les modalités de contrôle, des zones de pâture, de leur charge en bétail et de leur rotation.

\section{- Autres contraintes}

Les études, menées en République Centrafricaine et concernant l'élevage au sens le plus large, font apparaître diverses autres contraintes parmi lesquelles un certain nombre résulte 'des besoins fondamentaux des populations.

Par exemple, la mise en valeur pastorale est, tout comme l'activité agricole, étroitement dépendante des facilités de communication. La pénétration des axes routiers en zone d'élevage favorise la venue des sédentaires qui y installent villages et cultures. Il s'ensuit une cohabitation cultivateurs-éleveurs qui favorise les échanges, permet de satisfaire certains besoins réciproques et entraîne l'implantation de commerces. L'approvisionnement des populations en produits de base, souvent importés, s'en trouve facilité (sel, médicaments, articles manufacturés, certaines denrées alimentaires...) et l'accroissement des échanges commerciaux induit une certaine élévation du niveau de vie.

\section{Actions à entreprendre}

Du contexte de l'élevage en République Centrafricaine brièvement suggéré ci-dessus, il res- 
sort que les actions à entreprendre pour une amélioration de l'exploitation du potentiel pastoral des savanes de la zone sub-guinéenne se placent, simultanément, sur les plans de la recherche et du développement.

\section{- Programmes de recherches}

Les recherches concernant l'amélioration des méthodes d'exploitation des pâturages de savane, susceptibles d'orienter leur évolution floristique vers une « savanisation" accrue, ont une importance fondamentale.

Cependant, bien que les études intéressant les problèmes de l'exploitation des parcours, réalisées depuis 1960, aient fourni une importante somme de données qui constituent une base de travail solide, l'expérimentation en vraie grandeur qui, seule, apporterait aux praticiens la preuve du bien fondé des conclusions des travaux antérieurs, fait défaut.

Par suite, il serait judicieux de mettre en place un ou plusieurs modèles de systèmes d'exploitation, doublés de structures d'information et de vulgarisation auprès des éleveurs, afin de pouvoir préparer tous ceux qui vivent de l'élevage à la nécessaire rationalisation de leurs modes d'exploitation des parcours.

- Sur le plan fourrager, les programmes de recherches devraient mettre au point :

- les modes de découpage des terrains de parcours en parcelles de potentiels fourragers correspondants à la période d'utilisation et à l'importance du troupeau considéré. Ce parcellaire permettrait de pratiquer une pâture tournante capable d'assurer à la fois l'affouragement optimal du bétail et la lutte contre la dégradation de la végétation pastorale;

- les techniques de réalisation des pare-feu et d'emploi des feux de brousse qui permettront d'accorder les besoins alimentaires des animaux avec les périodes et les durées de pousse ou de repousse de la végétation pastorale et avecles variations de sa valeur alimentaire en fonction du stade des plantes pâturées;

- enfin, d'autres mesures un peu plus précises comme le respect des durées optimales des périodes de mise en repos de la végétation en fonction du milieu, de l'exploitation, de la composition floristique ou de la pression animale, pourraient être étudiées pour une vulgarisation plus lente.

- Sur le plan de la production animale - la question de la protection sanitaire du cheptel étant réglée - les recherches devront porter sur la mise au point de systèmes vulgarisables permettant d'optimiser la transformation de la production végétale en produits animaux; par exemple, la pâture des repousses les plus riches par des troupeaux de jeunes en croissance ou celle des stades végétaux plus pauvres par les animaux âgés, peut être préconisée.

Par suite de la diversité des contextes locaux (éloignement, débouchés, etc.) les méthodes devront être adaptées aux possibilités des milieux considérés (par exemple capacité d'intensification, de groupage des vêlages, de déstockage des jeunes mâles, etc...) et aux objectifs d'élevage les plus appropriés à la région (production de jeunes, de lait, d'animaux finis ou d'animaux de trait, etc...).

\section{- Programmes de développement}

L'amélioration des méthodes d'élevage et des niveaux de vie dans le secteur de l'élevage extensif semble, en grande partie, dépendre de l'ouverture de débouchés correspondant aux potentiels de production animale des zones d'élevage.

En premier lieu, la remise en état ou la création de voies de communication apparaissent urgentes. Des axes routiers nombreux facilitent les échanges commerciaux aussi bien que le transit du bétail vers les centres d'achat ou d'abattage et favorisent aussi la venue des sédentaires dans la zone pastorale et, par suite, le rapprochement des éleveurs et des agriculteurs. Cette cohabitation entraîne une certaine intégration, un accroissement des échanges et la possibilité de satisfaire des besoins plus nombreux. Il s'ensuit une motivation pour la vente des produits animaux dont la répercussion est une tendance à l'intensification des activités économiques dont la première, pour les éleveurs, est la production animale.

Sur un autre plan, la création d'établissements d'embouche à proximité des grandes agglomérations, tant pour les besoins de la consommation que pour l'exportation, peut motiver le développement de la commercialisation du bétail. Si une politique d'achat favorable aux éleveurs est instaurée, elle provoquera un accroissement de l'exploitation du cheptel.

Enfin, sur le plan humain, la création de centres d'approvisionnement en produits de base (médicamenteux et alimentaires en particulier) de dispensaires et d'écoles, dans la zone pastorale, en constituant le noyau des futurs villages peut catalyser la fixation définitive des 
éleveurs. Celle-cip̨ ' son tour, entraînera une diminution de la transhumance, une meilleure exploitation des parcours, si elle est accompagnée du cadre légal indispensable à la bonne utilisation permanente des pâturages par l'éleveur. Ce cadre est un code rural qui, complétant le " code d'élevage » que constitue la loi 65-61, définira avec le mode d'attribution des parcours et leurs limites, les droits et les devoirs des groupes sociaux attributaires.

\section{CONCLUSION}

L'utilisation optimale des ressources fourragères naturelles accompagnée de l'application de mesures propres à éviter la dégradation du potentiel pastoral, doit permettre de transformer l'élevage en une activité économique de production animale.

En effet, actuellement, pour les pasteurs Bororos, l'élevage est encore, en quelque sorte, davantage un mode de vie assurant la subsistance qu'une activité économique.
L'augmentation des besoins en viande du pays devrait inciter les éleveurs à évoluer vers une véritable spéculation. Ils en sont le plus souvent conscients et, s'ils ne sont encore que très peu nombreux à être passés d'une activité de "cueillette » à une production organisée c'est probablement qu'ils n'ont pas la certitude que ce changement leur sera profitable à long terme. Confrontés aux incertitudes de l'avenir ils conservent une attitude passive, illustrée, en particulier, par leur répugnance à investir, même seulement en travail, pour conserver ou faciliter la régénération d'un capital vegétal qui leur est pourtant indispensable et que leur mode d'exploitation dégrade.

L'amélioration des techniques pastorales doit leur être rapidement bénéfique au niveau individuel ou au moins familial. Dans le cas contraire, n'étant pas «chez eux » ils n'entreprendront même pas d'actions aussi simples que des mesures de protection du potentiel pastoral comme, par exemple, la pratique des feux contrôlés et échelonnés, ou l'application d'une rotation sommaire.

\title{
SUMMARY
}

\section{Savanna pasture management in the Central African Republic}

\begin{abstract}
Zebu cattle breeding was introduced in the Central African Republic fifty years ago and since then it has been expanding in a relatively favorable environment.

But this cattle increase has contributed to a considerable degradation of the grassland vegetation, and thus threatens the very existence of the herd.

After reviewing some of the problems met by this animal production, several research and development plans are described to save the potential of the cattle production in the Central African Republic.
\end{abstract}

\section{RESUMEN}

\section{La explotación de los pastos de sabana en la República Centroafricana}

Gracias a condiciones de medio relativamente favorables, la ganaderia de cebues introducida en la República Centroafricana hace cerca de un medio siglo se ha sumamente desarrollado. Pero el crecimiento del ganado al provocar una importante degradación de la vegetación pastoral pone en peligro hasta la existencia del rebaño. deria.

Se examinan algunas de las obligaciones haciendo fuerza en dicha gana-

Se proponen varias acciones que se necesitaria adoptar desde el punto de vista de la investigación y del desarrollo para intentar salvaguardar el potencial de la ganaderia vacuna en la República Centroafricana.

\section{BIBLIOGRAPHIE}

1. BOUDET (G.). Pâturages de la zone tropicale humide. Connaissances acquises et besoins en recherches complémentaires. Rev. Elev. Méd. vét. Pays trop., 1977, 30 (2) : 175-180.

2. PEYRE DE FABREGUES (B.), Dégradation des pâturages naturels de l'Ouest Centrafricain. Rapport de première mission. Maisons-Alfort, I. E. M. V. T., novembre 1975.38 p. + annexes.

3. PEYRE DE FABREGUES, (B.), CAPITAINE (P.).
Aménagements de pâturages dans l'Ouest Centrafricain. Orientations nouvelles de l'élevage bovin. Maisons-Alfort, I. E. M. V. T., février 1977, 123 p.

4. PEYRE DE FABREGUES (B.). Modernisation pastorale à l'Est de Bambari (E. C. A.). Maisons-Alfort, I. E. M. V. T. ; Paris, AGROTEC, mars 1978, 45 p.

5. R. C. A. Législation. Loi 65-61 du 3 juin 1965 portant réglementation de l'élevage en République Centrafricaine (5 titres-41 articles). 